\title{
Angiotensin converting enzyme gene polymorphism and respiratory muscle function in infants
}

\begin{tabular}{|r|l|}
\hline Journal: & Pediatric Pulmonology \\
\hline Manuscript ID: & PPUL-10-0005.R3 \\
\hline Wiley - Manuscript type: & Original Article \\
\hline Date Submitted by the & 08-Jun-2010 \\
\hline Complete List of Authors: & $\begin{array}{l}\text { Dimitriou, Gabriel; University of Patras Medical School, NICU, } \\
\text { Department of Pediatrics } \\
\text { Papakonstantinou, Despina; University of Patras Medical School, } \\
\text { NICU, Department of Pediatrics } \\
\text { Stavrou, Eleana; University of Patras Medical School, Department of } \\
\text { General Biology } \\
\text { Tzifas, Sotirios; University of Patras Medical School, NICU, } \\
\text { Department of Pediatrics } \\
\text { Vervenioti, Aggeliki; University of Patras Medical School, NICU, } \\
\text { Department of Pediatrics } \\
\text { Athanassiadou, Aglaia; University of Patras Medical School, } \\
\text { Department of General Biology } \\
\text { Mantagos, Stefanos; University of Patras Medical School, } \\
\text { Department of Pediatrics }\end{array}$ \\
\hline Keywords: & \begin{tabular}{l} 
ACE gene , respiratory muscles, pressure-time index, infants \\
\hline
\end{tabular} \\
\hline \hline
\end{tabular}

\section{S) ScholaroNE \\ Manuscript Central}


Title:

Angiotensin converting enzyme gene polymorphism and respiratory muscle function in infants

\section{Corresponding author:}

Dimitriou Gabriel, MD, PhD,

Neonatal Intensive Care Unit,

Department of Pediatrics,

University Hospital of Patras, 265 04, Rio, Patras, Greece,

Tel. (+30) 2610999856 ,

Fax. (+30) 2610994683 ,

email: gdimitriou@med.upatras.gr

\section{Authors}

1. Dimitriou Gabriel, MD, PhD, Associate Professor of Pediatrics-Neonatology, Neonatal Intensive Care Unit, Department of Pediatrics, University of Patras Medical School, Rio, Patras, Greece

2. Papakonstantinou Despina, MD, Research fellow, Neonatal Intensive Care Unit, Department of Pediatrics, University of Patras Medical School, Rio, Patras, Greece

3. Stavrou F. Eleana, MSC, Department of General Biology, University of Patras Medical School, Rio, Patras, Greece

4. Tzifas Sotirios, MD, Attending Neonatologist, Neonatal Intensive Care Unit, Department of Pediatrics, University of Patras Medical School, Rio, Patras, Greece 
5. Vervenioti Aggeliki, MD, Research Fellow, Neonatal Intensive Care Unit, Department of Pediatrics, University of Patras Medical School, Rio, Patras, Greece

6. Athanassiadou Aglaia, PhD, Professor of Biology-Molecular Genetics, Department of General Biology, University of Patras Medical School, Rio, Patras, Greece

7. Mantagos Stefanos MD, Professor of Pediatrics, Department of Pediatrics, University of Patras Medical School, Rio, Patras, Greece

\section{Meeting at which the paper presented}

$1^{\text {st }}$ International Congress of UENPS Global Neonatology \& Perinatology, 2008, Rome, Italy

\section{Key Words}

ACE gene, respiratory muscles, pressure-time index, infants

\section{Running Head}

ACE gene and respiratory muscle function

\section{Word count}

Abstract: 231; Text: 2888 


\begin{abstract}
Objective: Angiotensin converting enzyme (ACE) gene contains a polymorphism consisting of either the presence (I) or absence (D) of a 287 base pair fragment. Recent studies have suggested that the I-allele may be associated with superior exercise endurance; respiratory muscle function may be similarly influenced. The pressure-time index of inspiratory muscles (PTImus) is a measure of the loadcapacity ratio of the inspiratory muscles. The objective of this study was to determine whether infants homozygous for the I-allele have lower PTImus compared to infants homozygous for the D-allele or heterozygous I/D.
\end{abstract}

Patients and Methods: One hundred thirty two infants were studied. ACE genotyping was performed by polymerase chain reaction amplification, using DNA from peripheral blood. PTImus was calculated as $\left(\mathrm{Pi}_{\text {mean }} / \mathrm{Pi}\right.$ max $) \times(T i / T$ tot $)$, where $\mathrm{Pi}_{\text {mean }}$ was the mean inspiratory pressure estimated from airway pressure, generated 100 milliseconds after an occlusion $\left(\mathrm{P}_{0.1}\right), \mathrm{Pi}_{\max }$ was the maximum inspiratory pressure and $\mathrm{Ti} / \mathrm{T}$ tot was the ratio of the inspiratory time to the total respiratory cycle time. $\mathrm{Pi}_{\max }$ was the largest pressure generated during brief airway occlusions performed at the end of a spontaneous crying effort.

Results: Infants with I/I genotype had significantly lower PTImus than infants with either $D / D$ or $I / D$ genotypes ( $p=0.000007$ ). ACE genotype was significantly related $(p=0.005)$ to PTImus measurements, independent of other factors that may affect respiratory muscle function.

Conclusion: These results suggest that an association of ACE genotypes with PTImus measurements may exist in infants. 


\section{INTRODUCTION}

Angiotensin I-converting enzyme (ACE) converts angiotensin I to vasoactive and aldosterone-stimulating peptide angiotensin II and it is an important component of the local renin-angiotensin systems (RASs). ${ }^{1,2}$ There is a significant variation in circulating ACE activity among individuals, which may be genetically determined. The human ACE gene is located on chromosome 17 (17q23) and contains a two allele polymorphism, consisting of either the presence (insertion, I) or absence (deletion, D) of a 287 base pair (bp) fragment. ${ }^{3}$ The deletion is associated with increased ACE activity in both tissue and circulation and accounts for about $44 \%$ of the intra-individual variation in plasma ACE activity in Caucasians. ${ }^{3}$ Recent studies have suggested that I-allele may be associated with superior exercise endurance, ${ }^{4,5}$ partly through improved skeletal muscle metabolic efficiency. ${ }^{6}$ Respiratory muscle function may be similarly influenced.

Several methods have been used to assess the endurance properties of respiratory muscles. Diaphragmatic pressure-time index (PTIdi) is a measure of the load-capacity ratio of the diaphragm. ${ }^{7}$ In adults, a PTIdi greater than 0.15-0.18 may indicate impending diaphragm fatigue. ${ }^{7}$ Determination of PTIdi, however, is invasive, requiring the use of an esophageal catheter. A non-invasive method of measuring pressure-time index of inspiratory muscles (PTImus) has been validated in both adults $^{8}$ and infants. ${ }^{9}$

The aim of this study was to test the hypothesis that infants homozygous for the Iallele may have lower PTImus compared to infants homozygous for the D-allele or heterozygous I/D. 


\section{METHODS}

\section{Patients}

Infants cared for at the Neonatal Intensive Care Unit- Pediatric Department of the University General Hospital of Patras, Greece, were eligible for the study. Infants were entered into the study if parents gave informed written consent. The study was approved by the local Research Ethics Committee. Part of the studied population was recruited from a study examining the association of ACE genotypes and circulating blood ACE on diaphragmatic function in infants. All infants were studied awake, before discharge, in supine position and at least one hour after a feed. Infants had no respiratory symptoms for at least 3 days before measurement. Furthermore, infants were on full oral feeds, had serum electrolytes, calcium, magnesium and phosphates within normal range and did not receive any methylxanthines.

\section{ACE genotype determination}

ACE genotyping was performed on DNA extracted from whole blood, collected from an indwelling catheter or via peripheral venipuncture during routine blood sampling. The method has been previously described. ${ }^{10}$ Briefly, DNA was extracted from 200 $\mu$ l blood using Qiamp spin columns (Blood mini kit- Qiagen, QIAGEN Inc., Germantown, U.S.A). DNA was analyzed by electrophoresis on an agarose gel. DNA amplification of the $16^{\text {th }}$ ACE intron was performed using two sets of primers flanking the polymorphic site, (outer and inner primers), because mistyping of the $D / D$ genotype is reported to occur using the conventional amplification with insertion/deletion (I/D) flanking primer. ${ }^{11}$ 


\section{Measurement of PTImus}

Airflow was measured using a pneumotachograph (Mercury F10L, GM Instruments, Kilwinning, Scotland) connected to a differential pressure transducer (DP45, range \pm $2 \mathrm{~cm} \mathrm{H}_{2} \mathrm{O}$, Validyne Corp, Northridge, CA, USA). Airway pressure (Paw) was measured from a side port on the pneumotachograph using a differential pressure transducer (DP45, range $\pm 100 \mathrm{~cm} \mathrm{H} \mathrm{H}_{2} \mathrm{O}$, Validyne Corp, Northridge, CA, USA). The signals from the pressure transducers were amplified using a carrier amplifier (Validyne CD 280, Validyne Corp, Northridge, CA, USA). The pressure and flow signals were recorded and displayed in real time on a computer (Dell Optiplex GX620, Dell Inc., Texas, U.S.A) running Labview ${ }^{\mathrm{TM}}$ software (National Instruments, Austin, Texas, U.S.A) with analog-to-digital sampling at $100 \mathrm{~Hz}$ (16-bit NI PCl6036E, National Instruments, Austin, Texas, U.S.A).

$P_{0.1}$ was calculated as the airway pressure generated 100 milliseconds after an occlusion, using a face mask and a unidirectional valve which allowed expiration but not inspiration, while the infant was quietly breathing. At least four airway occlusions were performed and the average $P_{0.1}$ was calculated. Pressure-time index of the inspiratory muscles (PTImus), was calculated as: $\mathrm{PTImus}=\left(\mathrm{Pi}_{\text {mean }} / \mathrm{Pi}_{\max }\right) \times(\mathrm{Ti} / \mathrm{Ttot})$ where $\mathrm{Pi}_{\text {mean }}$ was the average airway pressure during inspiration, obtained from the formula $\mathrm{Pi}_{\text {mean }}=5 \times \mathrm{P}_{0.1} \times \mathrm{Ti}^{12} \mathrm{Pi}_{\max }$ was the maximum inspiratory airway pressure, Ti was the inspiration time and Ttot was the total time for each breath calculated from the airway flow signal. To measure $\mathrm{Pi}_{\max }$, a facemask (total deadspace, $4.5 \mathrm{~mL}$ ) was held firmly over the infant's nose and mouth and the airway was occluded at the end of a spontaneous crying effort using a unidirectional valve attached to the pneumotachograph, which allowed expiration, but not inspiration. The occlusion was 
maintained for at least four inspiratory efforts. At least three airway occlusions were performed and the maximum $\mathrm{Pi}_{\max }$ achieved for individual, was recorded.

\section{Statistical analysis}

Data was tested for normality using the Shapiro-Wilk and D'Agostino skewness tests. Differences between ACE genotype groups were assessed for statistical significance using the Kruskal-Wallis and Dunn's post-hoc non-parametric tests and Cramer's V test, as appropriate. Stepwise multiple regression analysis was performed to determine whether the polymorphism of the ACE gene is related to PTImus measurements, independently to gestational (GA) and postnatal (PNA) age, birthweight (BW), gender and support from mechanical ventilation.

Statistical analysis was performed using StatView 5.0 (SAS Institute, Inc., NC, USA), NCSS 2007 (NCSS, Utah, USA) and SPSS version 17 (SPSS Inc, Chicago, IL, USA).

\section{RESULTS}

Between January 2007 and August 2008 from the 532 infants admitted in the Neonatal Intensive Care Unit of the University of Patras, one hundred thirty two infants were included in the study. Fifty eight infants required ventilation in the initial stage of their illness. The characteristics of the study population are presented in table 1.

Eighteen infants (13.6\%) were homozygous for the I-allele (I/I), 41 (31.1\%) homozygous for the D-allele (D/D) and 73 infants (55.3\%) were heterozygous I/D. A Hardy-Weinberg equilibrium (HWE) calculation was performed to examine whether 
genotype frequencies remain constant in the studied population. Participants were in HWE (Chi square for HWE 2.613, $\mathrm{p}=0.106)$.

Overall, there were no significant differences in the characteristics of the infants with I/I, D/D and I/D ACE genotypes (table 2). Only four infants were oxygen dependent at 36 weeks postmenstrual age ( 1 infant with I/I, 2 with I/D and 1 with D/D ACE genotypes). Infants with I/I genotype had lower PTImus than infants with either D/D or I/D genotypes (Kruskal-Wallis, $p=0.000007$; Dunn's test, $z$-value $=3.58$, $p<0.01$ and $z$-value $=4.88, p<0.01$, respectively) (table 2), (figure). No difference in regards to PTImus values was found between infants with $D / D$ and I/D genotypes (Dunn's test, z-value $=1.39$, n.s). Analysis of the components of the PTImus has shown that only the ratio of $\mathrm{Pi}_{\text {mean }}$ to $\mathrm{Pi}_{\text {max }}$ was lower in infants with the $\mathrm{I} / \mathrm{I}$ genotype, compared to infants with either the $D / D$ or $I / D$ genotypes (Kruskal-Wallis, $p=$ 0.000005; Dunn's test, $z$-value $=3.33, \quad p<0.01$ and $z$-value $=4.91, \quad p<0.01$, respectively). Lower values of $\mathrm{Pi}_{\text {mean }}$ in infants with $\mathrm{I} / \mathrm{I}$ genotype compared to infants with either $D / D$ or $1 / D$ genotypes, were also observed (Kruskal-Wallis, $p=0.0009$; Dunn's test, $z$-value $=2.61, p<0.05$ and $z$-value $=3.73, p<0.01$, respectively) (table 2 ). $\mathrm{Pi}_{\text {max }}$ measurements were not statistically different between the three groups (table 2). Stepwise regression analysis revealed that ACE genotypes were significantly related to the PTImus measurements after logarithmic transformation, independent of GA, BW, PNA, gender and support from mechanical ventilation ( $p=0.005)$ (table 3).

In the subgroup of infants that never required any form of ventilatory support, 10 infants (13.5\%) were homozygous I/I, 20 (27.0\%) homozygous D/D and 44 infants (59.5\%) were heterozygous I/D. Infants in this subgroup were in HWE (Chi square for HWE 3.304, $p=0.069)$. No significant difference in the characteristics of the 
infants with I/I, D/D and I/D ACE genotypes, was found (table 4). Infants with I/I genotype had lower PTImus than infants with either D/D or I/D genotypes (KruskalWallis, $p=0.0022 ;$ Dunn's test, $z$-value $=3.07, p<0.01$ and $z$-value $=3.39, p<0.01$, respectively), (table 4). No difference regarding PTImus values was found between infants with D/D and I/D genotypes (Dunn's test, z-value $=0.0008$, n.s). The ratio of $\mathrm{Pi}_{\text {mean }}$ to $\mathrm{Pi}_{\text {max }}$ was lower in infants with the $\mathrm{I} / \mathrm{I}$ genotype, compared to infants with either the $D / D$ or I/D genotypes (Kruskal-Wallis, $p=0.0015$; Dunn's test, $z$-value= 2.95, $\mathrm{p}<0.01$ and $\mathrm{z}$-value $=3.58, \mathrm{p}<0.01$, respectively). $\mathrm{Pi}_{\max }$ measurements were not statistically different between the three groups (table 4), but $\mathrm{Pi}_{\text {mean }}$ was lower in infants with $\mathrm{I} / \mathrm{I}$ genotype, compared to infants with either $\mathrm{D} / \mathrm{D}$ or $\mathrm{I} / \mathrm{D}$ genotypes (Kruskal-Wallis, $p=0.003$; Dunn's test, $z$-value $=3.31, p<0.01$ and $z$-value $=3.00$, $p<0.01$, respectively). In this subgroup, forty six infants never required any oxygen supplementation; 6 infants (13.0\%) were homozygous I/I, $13(28.3 \%)$ homozygous D/D and 27 infants (58.7\%) were heterozygous I/D. Infants in the subgroup that never required any oxygen supplementation were in HWE (Chi square for HWE 1.872, $\mathrm{p}=0.171$ ). The median (range) of PTImus of the whole subgroup was 0.078 (0.028-0.119). Infants with I/I ACE genotype had lower PTImus than infants with either $\mathrm{D} / \mathrm{D}$ or $\mathrm{I} / \mathrm{D}$ genotypes [median (range), $0.040(0.028-0.083)$ versus 0.073 (0.052-119) and 0.080 (0.040-117), respectively; Kruskal-Wallis, $p=0.0075$; Dunn's test, $z$-value $=2.55, p<0.05$ and $z$-value $=3.11, p<0.01$, respectively]. The ratio of $\mathrm{Pi}_{\text {mean }}$ to $\mathrm{Pi}_{\text {max }}$ and $\mathrm{Pi}_{\text {mean }}$ were lower in infants with the $\mathrm{I} / \mathrm{l}$ ACE genotype, compared to infants with either D/D or I/D genotypes (Kruskal-Wallis, $p=0.0047$; Dunn's test, $z-$ value $=2.42, p<0.05$ and $z$-value $=3.27, p<0.01$, respectively and Kruskal-Wallis, $p=$ 0.019; Dunn's test, $z$-value $=2.46, p<0.05$ and $z$-value $=2.74, p<0.05$, respectively). 
In the subgroup of the infants who required ventilation in the initial stage of their illness, 8 infants (13.8\%) were homozygous I/I, 21 (36.2\%) homozygous D/D and 29 infants (50\%) were heterozygous I/D. Participants were in HWE (Chi square for HWE 0.1622, $p=0.687$ ). Infants with I/I ACE genotype had lower PTImus than infants with either $D / D$ or I/D genotypes (Kruskal-Wallis, $p=0.0024$; Dunn's test, $z$-value $=1.98$, $\mathrm{p}<0.05$ and $\mathrm{z}$-value $=3.37, \mathrm{p}<0.01$, respectively)

\section{DISCUSSION}

In this study, an association between ACE genotypes and PTImus measurements in infants, was demonstrated. Infants homozygous for the I-allele had lower PTImus values than infants homozygous for the D-allele and heterozygous I/D. No difference in regards to PTImus values was found between infants with D/D and I/D genotypes. Furthermore, the ACE polymorphism was related to the PTImus measurements, independent of other factors, which could affect respiratory muscle performance. The association between ACE genotypes and PTImus measurements was replicated on the subgroup of infants that required ventilation in the initial stage of their illness and on the subgroup of infants that never required any form of ventilatory support.

One limitation of our study was the fact that measurements in an age-matched control group, consisting of infants in the postnatal ward, were not performed. The recruitment of such babies is extremely difficult. To overcome this issue, we measured infants in the neonatal unit that are free of factors that could possibly interfere with respiratory muscle function such as requirement of respiratory support. 
One part of the whole population consisted of infants that never required oxygen supplementation, indeed, and a separate analysis was performed in this subgroup.

Fatigue of respiratory muscles may result in an inability to maintain adequate alveolar ventilation and respiratory failure. A simple method of studying the endurance properties of inspiratory muscles, is the measurement of the duration that a specific submaximal task (maximum sustainable ventilation, external mechanical load) can be maintained (time limit or endurance time, TLim), referred to as the point where the inspiratory muscles failed to maintain the task, despite maximal effort. ${ }^{7}$ However, this kind of test is difficult to be applied to infants. Diaphragmatic pressuretime index (PTIdi), the product of the ratio of mean inspiratory transdiaphragmatic pressure per breath $\left(\mathrm{Pdi}_{\text {mean }}\right)$ to maximum transdiaphragmatic pressure $\left(\mathrm{Pdi}_{\max }\right)$ and the ratio of the inspiratory time ( $\mathrm{Ti}$ ) to the total respiratory cycle time (Ttot), is closely related to the Tlim. ${ }^{13}$ The determination of PTIdi, however, is rather invasive, since it requires the placement of an esophageal catheter. Analysis of inspiratory muscle function by measurement of PTImus was first described by Gaultier et al. ${ }^{12}$ As measurement of $P_{01}$ is non-invasive, measurement of PTImus has the advantage of being relatively easy to be performed. This index was validated in adults. ${ }^{8}$ Studies in healthy children, demonstrated that PTImus ranged between 0.036 and $0.090 .{ }^{14,15}$ In spontaneously breathing infants, PTImus was compared to PTIdi and an agreement between the two methods, using Bland and Altman analysis, was found. ${ }^{9}$ In this study, PTImus measurements in infants that never required any oxygen supplementation ranged between 0.028 and 0.119 , something that is close to the values observed in healthy children. 
Several studies have suggested that there is an association of the ACE I/D polymorphism with exercise performance. British Army recruits were examined before and after an identical 10-week general physical training program. ${ }^{5}$ Although, pre-training performance was independent of genotype, the improvement in performance was 11 -fold greater among those of $\mathrm{I} / \mathrm{I}$, compared with $\mathrm{D} / \mathrm{D}$ genotype. ${ }^{5}$ Consistently, a significant excess of the $\mathrm{l} / \mathrm{I}$ genotype was found in Australian national rowers attending their pre-Olympics selection trial, compared to matched healthy controls. ${ }^{16}$ The frequency of I-allele was higher in British Olympic-standard distance runners. ${ }^{4}$ Furthermore, genotype distribution and I-allele frequency differed significantly between elite mountaineers, who had ascended beyond $7000 \mathrm{~m}$ without the use of supplemental oxygen and controls. ${ }^{5}$ All such studies are prone to difficulties in comparison, as the definition of the "elite athlete" and the heterogeneity of the elite athlete phenotype may be variable. The polymorphism of the ACE gene has been examined in infants, but not in association with muscle endurance. ${ }^{17,18}$

The association of the ACE genotype and respiratory muscle function, has not been widely examined yet. In a study of COPD (Chronic Obstructive Pulmonary Disease) patients and healthy control subjects, the ACE genotype was related to the regulation of skeletal muscle aerobic work efficiency, but it was not associated with the ventilatory responses to exercise. ${ }^{19}$ The relationship between circulating ACE activity, ACE I/D genotype, mechanical efficiency and the maximal rate of oxygen uptake has been assessed in untrained women. ${ }^{20}$ Neither circulating ACE activity nor ACE genotype showed a statistically significant association with any of the endurance phenotypes measured. However, ACE inhibition in heart failure increases respiratory muscle strength ${ }^{21}$ and exercise tolerance..$^{22,23}$ 
The enhanced endurance performance in the ACE I/I genotype may be due to higher oxygen delivery, conversion of fast-twitch, type II, to slow-contracting, type I, muscle fibers, increased mitochondrial numbers and density, and raised myoglobin content of the muscle. ${ }^{24}$ ACE I-allele compared to D-allele is associated with an increased percentage of fatigue-resistant type I skeletal muscle fibres ${ }^{25}$ and hence with enhanced mechanical ${ }^{6}$ and metabolic $^{26}$ efficiency of the trained muscle. The effect of ACE genotype on energy balance, might be mediated through several different pathways, such as systemic endocrine or nutritional mechanisms. ${ }^{26}$ Local RASs exist in several tissues. The adipose RAS may alter mobilization of substrate from fat stores and affect metabolism. ${ }^{26}$ Finally, the local skeletal muscle RAS may modify the use of substrate and enhance the metabolic efficiency of the muscles. ${ }^{26}$

Several factors may affect respiratory muscle endurance such as nutrition, ${ }^{27}$ prolonged ventilatory support, ${ }^{28}$ drugs, ${ }^{29,30,31}$ as well as phosphate, ${ }^{32}$ calcium $^{33}$ and magnesium ${ }^{34}$ blood levels. All infants were measured prior to discharge, being on full enteral feeds, they did not receive any medication and their biochemistry blood tests were within the normal range during measurements.

PTImus integrates all components that may affect inspiratory muscles while breathing at rest, i.e, timing of breathing cycle, inspiratory demand at rest and maximal inspiratory force reserve. When we considered these components, the timing of the breath cycle (Ti/Ttot) and the maximal inspiratory force reserve $\left(\mathrm{Pi}_{\max }\right)$ were not statistically different between infants with I/I, D/D and I/D ACE genotype. However, the $\mathrm{Pi}_{\text {mean }} / \mathrm{Pi}_{\max }$ ratio and the $\mathrm{Pi}_{\text {mean }}$ (inspiratory demand at rest), were lower in infants with the $\mathrm{I} / \mathrm{I}$ compared with infants with $\mathrm{D} / \mathrm{D}$ and $\mathrm{I} / \mathrm{D}$ genotypes. $A$ higher inspiratory demand at rest $\left(\mathrm{Pi}_{\text {mean }}\right)$ in infants with $\mathrm{D} / \mathrm{D}$ and $\mathrm{I} / \mathrm{D}$, compared with 
infants with I/I ACE genotypes, could suggest that a higher elastic and/or resistive load (existing lung disease) imposed on the respiratory muscles of infants with I/D and D/D genotypes compared with infants with I/I ACE genotypes, may exist. Indeed, infants with non-I/I ACE genotypes have been suggested to be more likely to develop bronchopulmonary dysplasia, perhaps as a consequence of the role of ACE activity in the inflammatory cascade. ${ }^{17}$ However, in this study, no difference in regards to bronchopulmonary dysplasia rate between infants with I/I, I/D and D/D ACE genotypes was noted. In addition, the same association between ACE genotypes and $\mathrm{Pi}_{\text {mean }}$ was replicated in a subgroup of infants that never required any form of respiratory support and in infants that never required any oxygen supplementation.

Fatigue threshold of inspiratory muscles has not yet been established in infants. All infants measured, were spontaneously breathing on air. Therefore, the PTImus results of all infants would be expected to lie far from the fatigue threshold. These results, however, indicate an increased effectiveness of inspiratory muscles of infants with the I// ACE genotype, due to a lower energy demand and thus to a lower risk of respiratory muscle fatigue, under conditions that increase inspiratory load. ${ }^{35,36}$ The findings of this study suggest that in clinical practice, infants with $1 / /$ ACE genotype, if supported by mechanical ventilation, may succeed extubation more often than infants with I/D and D/D ACE genotypes or may have a lower risk of respiratory failure under conditions that increase inspiratory load, such as respiratory infections.

In conclusion, these results suggest that an association of ACE genotypes with 
PTImus measurements may exist in infants, independent of other factors, which could affect respiratory muscle function.

\section{ACKNOWLEDGMENTS}

None

\section{COMPETING INTERESTS}

The authors declare that they have no financial or other relationships that might lead to a conflict of interest.

\section{FUNDING}

None

\section{ETHICS COMMITTEE APPROVAL}

The study was approved by the Ethics Committee of the University Hospital of Patras, Patras, Greece 


\section{REFERENCES}

1. Woods DR, Pollard AJ, Collier DJ, Jamshidi Y, Vassiliou V, Hawe E, Humphries SE, Montgomery HE. Insertion/deletion polymorphism of the angiotensin I-converting enzyme gene and arterial oxygen saturation at high altitude. Am J Respir Crit Care Med 2002;166(3):362-366.

2. Jones A, Woods DR. Skeletal muscle RAS and exercise performance. Int J Biochem Cell Biol 2003;35(6):855-866.

3. Tiret L, Rigat B, Visvikis S, Breda C, Corvol P, Cambien F, Soubrier F. Evidence, from combined segregation and linkage analysis, that a variant of the angiotensin l-converting enzyme (ACE) gene controls plasma ACE levels. Am J Hum Genet 1992;51(1):197-205.

4. Myerson S, Hemingway H, Budget R, Martin J, Humphries S, Montgomery $\mathrm{H}$. Human angiotensin I-converting enzyme gene and endurance performance. J Appl Physiol 1999;87(4):1313-1316.

5. Montgomery HE, Marshall R, Hemingway H, Myerson S, Clarkson P, Dollery C, Hayward M, Holliman DE, Jubb M, World M, Thomas EL, Brynes AE, Saeed N, Barnard M, Bell JD, Prasad K, Rayson M, Talmud PJ, Humphries SE. Human gene for physical performance. Nature 1998;393(6682):221-222.

6. Williams AG, Rayson MP, Jubb M, World M, Woods DR, Hayward M, Martin J, Humphries SE, Montgomery HE. The ACE gene and muscle performance. Nature 2000;403(6770):614.

7. ATS/ERS Statement on respiratory muscle testing. Am J Respir Crit Care Med 2002;166(4):518-624.

8. Ramonatxo M, Boulard P, Prefaut C. Validation of a noninvasive tension-time index of inspiratory muscles. J Appl Physiol 1995;78(2):646-653. 
9. Dimitriou G, Patel S, Patel J, Rafferty GF, Greenough A. A non-invasive tension-time index of respiratory muscles in neonates. European Respiratory Society Annual Congress. Glasgow, UK; 2004.

10. Lindpaintner K, Pfeffer MA, Kreutz R, Stampfer MJ, Grodstein F, LaMotte F, Buring J, Hennekens $\mathrm{CH}$. A prospective evaluation of an angiotensinconverting-enzyme gene polymorphism and the risk of ischemic heart disease. N Engl J Med 1995;332(11):706-711.

11. Shanmugam V, Sell KW, Saha BK. Mistyping ACE heterozygotes. PCR Methods Appl 1993;3(2):120-121.

12. Gaultier C, Boule M, Tournier G, Girard F. Inspiratory force reserve of the respiratory muscles in children with chronic obstructive pulmonary disease. Am Rev Respir Dis 1985;131(6):811-815.

13. Bellemare F, Grassino A. Effect of pressure and timing of contraction on human diaphragm fatigue. J Appl Physiol 1982;53(5):1190-1195.

14. Hayot M, Guillaumont S, Ramonatxo M, Voisin M, Prefaut C. Determinants of the tension-time index of inspiratory muscles in children with cystic fibrosis. Pediatr Pulmonol 1997;23(5):336-343.

15. Hahn A, Ankermann T, Claass A, Mann M, Lindemann H, Neubauer BA. Noninvasive tension time index in relation to severity of disease in children with cystic fibrosis. Pediatr Pulmonol 2008;43(10):973-981.

16. Gayagay G, Yu B, Hambly B, Boston T, Hahn A, Celermajer DS, Trent RJ. Elite endurance athletes and the ACE I allele--the role of genes in athletic performance. Hum Genet 1998;103(1):48-50. 
17. Kazzi SN, Quasney MW. Deletion allele of angiotensin-converting enzyme is associated with increased risk and severity of bronchopulmonary dysplasia. J Pediatr 2005;147(6):818-822.

18. Harding D, Dhamrait S, Marlow N, Whitelaw A, Gupta S, Humphries S, Montgomery $\mathrm{H}$. Angiotensin-converting enzyme DD genotype is associated with worse perinatal cardiorespiratory adaptation in preterm infants. J Pediatr 2003;143(6):746-749.

19. Zhang X, Wang C, Dai H, Lin Y, Zhang J. Association between angiotensinconverting enzyme gene polymorphisms and exercise performance in patients with COPD. Respirology 2008;13(5):683-688.

20. Day SH, Gohlke P, Dhamrait SS, Williams AG. No correlation between circulating ACE activity and VO2max or mechanical efficiency in women. Eur J Appl Physiol 2007;99(1):11-18.

21. Coirault C, Hagege A, Chemla D, Fratacci MD, Guerot C, Lecarpentier Y. Angiotensin-converting enzyme inhibitor therapy improves respiratory muscle strength in patients with heart failure. Chest 2001;119(6):1755-1760.

22. Mancini DM, Davis L, Wexler JP, Chadwick B, LeJemtel TH. Dependence of enhanced maximal exercise performance on increased peak skeletal muscle perfusion during long-term captopril therapy in heart failure. Journal of the American College of Cardiology 1987;10(4):845-850.

23. Drexler $H$, Banhardt $U$, Meinertz $T$, Wollschlager $H$, Lehmann $M$, Just $H$. Contrasting peripheral short-term and long-term effects of converting enzyme inhibition in patients with congestive heart failure. A double-blind, placebocontrolled trial. Circulation 1989;79(3):491-502. 
24. Bloom SR, Johnson RH, Park DM, Rennie MJ, Sulaiman WR. Differences in the metabolic and hormonal response to exercise between racing cyclists and untrained individuals. J Physiol 1976;258(1):1-18.

25. Zhang B, Tanaka H, Shono N, Miura S, Kiyonaga A, Shindo M, Saku K. The I allele of the angiotensin-converting enzyme gene is associated with an increased percentage of slow-twitch type I fibers in human skeletal muscle. Clin Genet 2003;63(2):139-144.

26. Montgomery H, Clarkson P, Barnard M, Bell J, Brynes A, Dollery C, Hajnal J, Hemingway $H$, Mercer D, Jarman P, Marshall R, Prasad K, Rayson M, Saeed N, Talmud $\mathrm{P}$, Thomas L, Jubb M, World M, Humphries S. Angiotensinconverting-enzyme gene insertion/deletion polymorphism and response to physical training. Lancet 1999;353(9152):541-545.

27. Arora NS, Rochester DF. Effect of body weight and muscularity on human diaphragm muscle mass, thickness, and area. J Appl Physiol 1982;52(1):6470.

28. Tobin MJ, Alex C. Discontinuation of mechanical ventilation. In: Tobin M, editor. Principles and Practice of Mechanical Ventilation. New York: McGrawHill; 1994. p 1177-1206.

29. Aubier M, Murciano D, Menu Y, Boczkowski J, Mal H, Pariente R. Dopamine effects on diaphragmatic strength during acute respiratory failure in chronic obstructive pulmonary disease. Ann Intern Med 1989;110(1):17-23.

30. Vires N, Aubier M, Murciano D, Fleury B, Talamo C, Pariente R. Effects of aminophylline on diaphragmatic fatigue during acute respiratory failure. Am Rev Respir Dis 1984;129(3):396-402. 
31. Supinski GS, Levin S, Kelsen SG. Caffeine effect on respiratory muscle endurance and sense of effort during loaded breathing. J Appl Physiol 1986;60(6):2040-2047.

32. Aubier M, Murciano D, Lecocguic $Y$, Viires N, Jacquens $Y$, Squara $P$, Pariente R. Effect of hypophosphatemia on diaphragmatic contractility in patients with acute respiratory failure. N Engl J Med 1985;313(7):420-424.

33. Aubier M, Viires N, Piquet J, Murciano D, Blanchet F, Marty C, Gherardi R, Pariente R. Effects of hypocalcemia on diaphragmatic strength generation. J Appl Physiol 1985;58(6):2054-2061.

34. Dhingra S, Solven F, Wilson A, McCarthy DS. Hypomagnesemia and respiratory muscle power. Am Rev Respir Dis 1984;129(3):497-498.

35. Roussos C, Macklem P. Inspiratory muscle fatigue. In: Macklem P, Mead J, editors. The Handbook of Physiology The Respiratory System. Volume III. Bethesda, MD: American Physiological Society; 1986. p 511-527.

36. Vassilakopoulos T, Roussos C. Neuromuscular respiratory failure. In: Slutsky A, Takala R, Torres R, editors. Clinical Critical Care Medicine. St. Louis: Mosby; 2006. p 275-282. 
Figure legend

Comparison of PTImus results of infants with I/I $(n=18), I / D(n=73)$ and $D / D(n=41)$ ACE genotypes. Box and whisker plot: fine horizontal lines represent $10^{\text {th }}, 25^{\text {th }}, 50^{\text {th }}$, $75^{\text {th }}$ and $90^{\text {th }}$ centiles of PTImus. Outliers are plotted as discrete data points. 
Table 1. Characteristics of the study population. Data are demonstrated as $n(\%)$ or median (range).

Number of studied infants

Gestational age (weeks)

Birth weight $(g)$

Gender (male)

Preterm

Duration of mechanical ventilation (days)

Diagnoses on admission

Respiratory distress syndrome (RDS)

Transient tachypnea of the newborn (TTN)

Meconium aspiration syndrome

Infection

Congenital pneumonia

Prematurity (low birth weight)

Birth depression

Intrauterine growth retardation

At the time of measurement

Postnatal age (days)

Postmenstrual age (PMA) (weeks)

Preterm (PMA<37 weeks)

Weight (g)
132

$35.5 \quad(27-40)$

2680 (900-4540)

$76 \quad$ (57.6)

$80 \quad(60.6)$

$0 \quad(0-59)$

$3 \quad$ (2.3)

$9 \quad(1-107)$

$37.1 \quad(32.4-46.7)$

$61 \quad(46.2)$

2550 (1850-4610) 
Table 2. Characteristics and PTImus results of infants with $I / I, I / D$ and $D / D$ ACE genotypes. Data are demonstrated as n (\%) or median (range in parentheses).

\begin{tabular}{|c|c|c|c|c|}
\hline \multirow[b]{2}{*}{ ACE genotypes (n) } & \multirow[b]{2}{*}{ II (18) } & \multirow[b]{2}{*}{ ID (73) } & \multirow[b]{2}{*}{ DD (41) } & \multirow[t]{2}{*}{$p$ value } \\
\hline & & & & \\
\hline \multicolumn{5}{|l|}{ Gestational age } \\
\hline (weeks) & $36(27-40)$ & $35(28-40)$ & $36(29-40)$ & $0.648^{\mathrm{a}}$ \\
\hline \multirow[t]{2}{*}{ Birth weight (g) } & 2780 & 2700 & 2580 & \\
\hline & $(960-4150)$ & $(900-4080)$ & $(1070-4540)$ & $0.598^{\mathrm{a}}$ \\
\hline Male gender & $8(44.4 \%)$ & $42(57.5 \%)$ & $26(63.4 \%)$ & $0.118^{b}$ \\
\hline \multicolumn{5}{|l|}{ Duration of mechanical } \\
\hline ventilation (days) & $0(0-59)$ & $0(0-21)$ & $1(0-8)$ & $0.857^{\mathrm{a}}$ \\
\hline \multicolumn{5}{|c|}{ At the time of measurement } \\
\hline Postnatal age (days) & $8.5(1-107)$ & $9(1-62)$ & $9(1-56)$ & $0.692^{a}$ \\
\hline \multirow[t]{2}{*}{ Weight $(\mathrm{kg})$} & 2660 & 2560 & 2450 & \\
\hline & $(1850-4050)$ & $(1850-4140)$ & $(1880-4610)$ & $0.471^{\mathrm{a}}$ \\
\hline \multirow[t]{2}{*}{ PTImus } & 0.046 & 0.077 & 0.071 & \\
\hline & $(0.023-0.085)$ & $(0.040-0.170)$ & $(0.036-0.119)$ & $0.000007^{\mathrm{a}}$ \\
\hline $\mathrm{Ti} / \mathrm{Tot}$ & $0.39(0.27-0.58)$ & $0.43(0.31-0.55)$ & $0.45(0.35-0.51)$ & $0.081^{a}$ \\
\hline \multirow[t]{2}{*}{$\mathrm{Pi}_{\text {mean }} / \mathrm{Pi}_{\max }$} & 0.118 & 0.182 & 0.160 & \\
\hline & $(0.052-0.171)$ & $(0.096-0.373)$ & $(0.086-0.322)$ & $0.000005^{a}$ \\
\hline \multirow[t]{2}{*}{$\mathrm{Pi}_{\text {mean }}\left(\mathrm{cmH}_{2} \mathrm{O}\right)$} & 7.77 & 10.18 & 9.98 & \\
\hline & $(3.71-13.48)$ & $(5.86-19.21)$ & $(4.48-17.64)$ & $0.0009^{a}$ \\
\hline \multirow[t]{2}{*}{$\mathrm{Pi}_{\max }\left(\mathrm{cmH}_{2} \mathrm{O}\right)$} & 66.9 & 58.6 & 62.8 & \\
\hline & $(46.8-88.7)$ & $(32.3-97.4)$ & $(28.0-91.8)$ & $0.271^{\mathrm{a}}$ \\
\hline
\end{tabular}

${ }^{a}$ Kruskal-Wallis test ${ }^{b}$ Cramer's V test 
Table 3. Results of stepwise regression analysis

\section{Partial Correlation}

Coefficient t Value Significance

\section{Dependent variable: PTImus}

ACE gene polymorphism

0.245

2.870

0.005

Birth weight

$-0.176$

$-2.035$

0.044

Gestational age

0.011

0.125

0.901

Postnatal age

0.020

0.222

0.825

Gender

$-0.033$

$-0.376$

0.708

Duration of mechanical

ventilation

$-0.061$

$-0.694$

0.489 
Table 4. Characteristics and PTImus results of infants with $I / I, I / D$ and $D / D$ ACE genotypes that never required respiratory support. Data are demonstrated as $\mathrm{n}(\%)$ or median (range in parentheses).

\begin{tabular}{|c|c|c|c|c|}
\hline \multirow[b]{2}{*}{ ACE genotypes (n) } & \multirow[b]{2}{*}{ II (10) } & \multirow[b]{2}{*}{ ID (44) } & \multirow[b]{2}{*}{$\mathrm{DD}(20)$} & \multirow[t]{2}{*}{$p$ value } \\
\hline & & & & \\
\hline \multicolumn{5}{|l|}{ Gestational age } \\
\hline (weeks) & $36(32-39)$ & $35.5(30-40)$ & $36.5(30-40)$ & $0.946^{\mathrm{a}}$ \\
\hline \multirow[t]{2}{*}{ Birth weight (g) } & 2780 & 2725 & 2740 & \\
\hline & $(1350-3650)$ & $(1130-4080)$ & $(1070-4540)$ & $0.982^{a}$ \\
\hline Male gender & $5(50 \%)$ & $26(59.1 \%)$ & $12(60 \%)$ & $0.065^{b}$ \\
\hline \multicolumn{5}{|c|}{ At the time of measurement } \\
\hline Postnatal age (days) & $4(1-26)$ & $6(1-39)$ & $8.5(1-41)$ & $0.146^{\mathrm{a}}$ \\
\hline \multirow[t]{2}{*}{ Weight (kg) } & 2675 & 2620 & 2685 & \\
\hline & $(1950-3500)$ & $(1890-4140)$ & $(1880-4610)$ & $0.889^{a}$ \\
\hline \multirow[t]{2}{*}{ PTImus } & 0.043 & 0.076 & 0.074 & \\
\hline & $(0.028-0.085)$ & $(0.040-0.117)$ & $(0.052-0.119)$ & $0.0022^{\mathrm{a}}$ \\
\hline \multirow[t]{2}{*}{$\mathrm{Ti} / \mathrm{Tot}$} & 0.39 & 0.43 & 0.46 & \\
\hline & $(0.27-0.51)$ & $(0.34-0.55)$ & $(0.35-0.51)$ & $0.058^{a}$ \\
\hline \multirow[t]{2}{*}{$\mathrm{Pi}_{\text {mean }} / \mathrm{Pi}_{\max }$} & 0.118 & 0.177 & 0.163 & \\
\hline & $(0.092-0.171)$ & $(0.096-0.336)$ & $(0.116-0.306)$ & $0.0015^{a}$ \\
\hline \multirow[t]{2}{*}{$\mathrm{Pi}_{\text {mean }}\left(\mathrm{cmH}_{2} \mathrm{O}\right)$} & 7.77 & 9.70 & 10.25 & \\
\hline & $(4.68-10.13)$ & $(6.20-19.21)$ & $(7.39-16.07)$ & $0.003^{a}$ \\
\hline \multirow[t]{2}{*}{$\mathrm{Pi}_{\max }\left(\mathrm{cmH}_{2} \mathrm{O}\right)$} & 61.4 & 58.6 & 63.3 & \\
\hline & $(46.8-88.7)$ & $(36.9-97.4)$ & $(32.7-91.8)$ & $0.637^{\mathrm{a}}$ \\
\hline
\end{tabular}

${ }^{a}$ Kruskal-Wallis test ${ }^{b}$ Cramer's $V$ test 

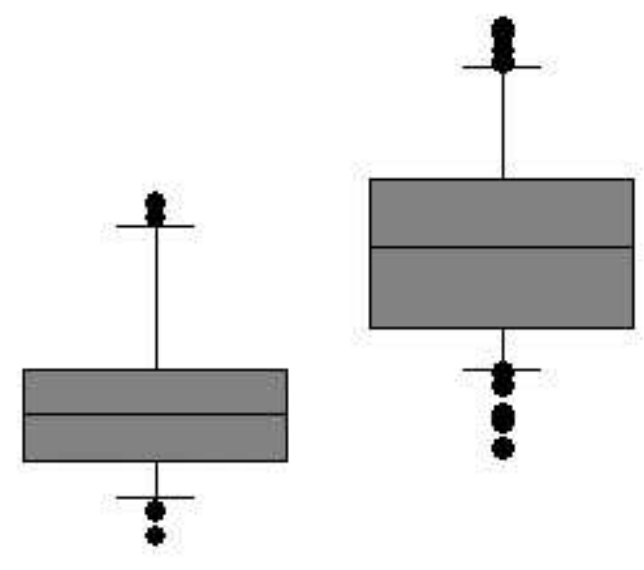

I/I
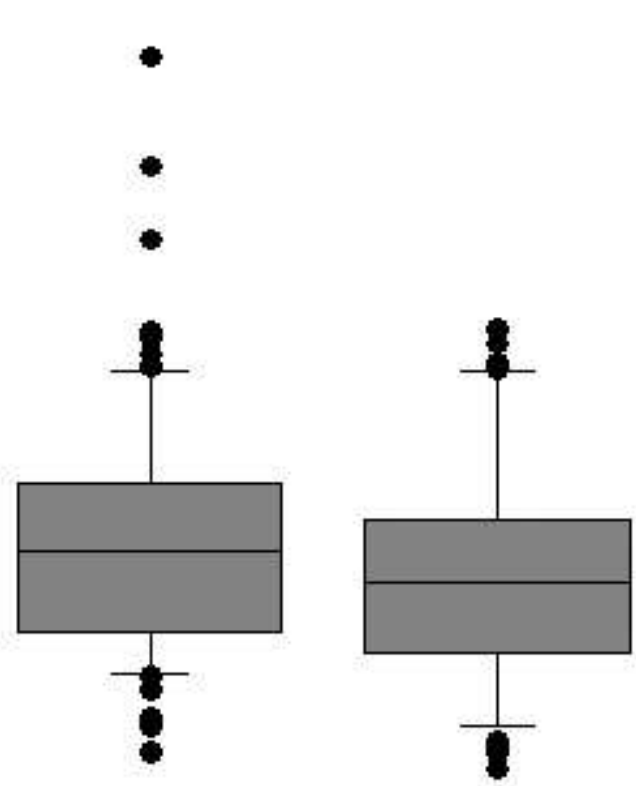

Figure

$39 \times 36 \mathrm{~mm}(300 \times 300$ DPI $)$

John Wiley \& Sons, Inc. 
Title:

Angiotensin converting enzyme gene polymorphism and respiratory muscle function in infants

\section{Corresponding author:}

Dimitriou Gabriel, MD, PhD,

Neonatal Intensive Care Unit,

Department of Pediatrics,

University Hospital of Patras, 265 04, Rio, Patras, Greece,

Tel. (+30) 2610999856 ,

Fax. (+30) 2610994683 ,

email: gdimitriou@med.upatras.gr

\section{Authors}

1. Dimitriou Gabriel, MD, PhD, Associate Professor of Pediatrics-Neonatology, Neonatal Intensive Care Unit, Department of Pediatrics, University of Patras Medical School, Rio, Patras, Greece

2. Papakonstantinou Despina, MD, Research fellow, Neonatal Intensive Care Unit, Department of Pediatrics, University of Patras Medical School, Rio, Patras, Greece

3. Stavrou F. Eleana, MSC, Department of General Biology, University of Patras Medical School, Rio, Patras, Greece

4. Tzifas Sotirios, MD, Attending Neonatologist, Neonatal Intensive Care Unit, Department of Pediatrics, University of Patras Medical School, Rio, Patras, Greece 
5. Vervenioti Aggeliki, MD, Research Fellow, Neonatal Intensive Care Unit, Department of Pediatrics, University of Patras Medical School, Rio, Patras, Greece

6. Athanassiadou Aglaia, PhD, Professor of Biology-Molecular Genetics, Department of General Biology, University of Patras Medical School, Rio, Patras, Greece

7. Mantagos Stefanos MD, Professor of Pediatrics, Department of Pediatrics, University of Patras Medical School, Rio, Patras, Greece

\section{Meeting at which the paper presented}

$1^{\text {st }}$ International Congress of UENPS Global Neonatology \& Perinatology, 2008, Rome, Italy

\section{Key Words}

ACE gene, respiratory muscles, pressure-time index, infants

\section{Running Head}

ACE gene and respiratory muscle function

\section{Word count}

Abstract: 231; Text: 2888 


\begin{abstract}
Objective: Angiotensin converting enzyme (ACE) gene contains a polymorphism consisting of either the presence (I) or absence (D) of a 287 base pair fragment. Recent studies have suggested that the I-allele may be associated with superior exercise endurance; respiratory muscle function may be similarly influenced. The pressure-time index of inspiratory muscles (PTImus) is a measure of the loadcapacity ratio of the inspiratory muscles. The objective of this study was to determine whether infants homozygous for the I-allele have lower PTImus compared to infants homozygous for the D-allele or heterozygous I/D.
\end{abstract}

Patients and Methods: One hundred thirty two infants were studied. ACE genotyping was performed by polymerase chain reaction amplification, using DNA from peripheral blood. PTImus was calculated as $\left(\mathrm{Pi}_{\text {mean }} / \mathrm{Pi}_{\max }\right) \times(\mathrm{Ti} / \mathrm{Tt}$ tot $)$, where $\mathrm{Pi}_{\text {mean }}$ was the mean inspiratory pressure estimated from airway pressure, generated 100 milliseconds after an occlusion $\left(\mathrm{P}_{0.1}\right), \mathrm{Pi}_{\max }$ was the maximum inspiratory pressure and $\mathrm{Ti} / \mathrm{T}$ tot was the ratio of the inspiratory time to the total respiratory cycle time. $\mathrm{Pi}_{\max }$ was the largest pressure generated during brief airway occlusions performed at the end of a spontaneous crying effort.

Results: Infants with I/I genotype had significantly lower PTImus than infants with either $D / D$ or $I / D$ genotypes ( $p=0.000007$ ). ACE genotype was significantly related $(p=0.005)$ to PTImus measurements, independent of other factors that may affect respiratory muscle function.

Conclusion: These results suggest that an association of ACE genotypes with PTImus measurements may exist in infants. 


\section{INTRODUCTION}

Angiotensin I-converting enzyme (ACE) converts angiotensin I to vasoactive and aldosterone-stimulating peptide angiotensin II and it is an important component of the local renin-angiotensin systems (RASs). ${ }^{1,2}$ There is a significant variation in circulating ACE activity among individuals, which may be genetically determined. The human ACE gene is located on chromosome 17 (17q23) and contains a two allele polymorphism, consisting of either the presence (insertion, I) or absence (deletion, D) of a 287 base pair (bp) fragment. ${ }^{3}$ The deletion is associated with increased ACE activity in both tissue and circulation and accounts for about $44 \%$ of the intra-individual variation in plasma ACE activity in Caucasians. ${ }^{3}$ Recent studies have suggested that I-allele may be associated with superior exercise endurance, ${ }^{4,5}$ partly through improved skeletal muscle metabolic efficiency. ${ }^{6}$ Respiratory muscle function may be similarly influenced.

Several methods have been used to assess the endurance properties of respiratory muscles. Diaphragmatic pressure-time index (PTIdi) is a measure of the load-capacity ratio of the diaphragm. ${ }^{7}$ In adults, a PTIdi greater than 0.15-0.18 may indicate impending diaphragm fatigue. ${ }^{7}$ Determination of PTIdi, however, is invasive, requiring the use of an esophageal catheter. A non-invasive method of measuring pressure-time index of inspiratory muscles (PTImus) has been validated in both adults $^{8}$ and infants. ${ }^{9}$

The aim of this study was to test the hypothesis that infants homozygous for the Iallele may have lower PTImus compared to infants homozygous for the D-allele or heterozygous I/D. 


\section{METHODS}

\section{Patients}

Infants cared for at the Neonatal Intensive Care Unit- Pediatric Department of the University General Hospital of Patras, Greece, were eligible for the study. Infants were entered into the study if parents gave informed written consent. The study was approved by the local Research Ethics Committee. Part of the studied population was recruited from a study examining the association of ACE genotypes and circulating blood ACE on diaphragmatic function in infants. All infants were studied awake, before discharge, in supine position and at least one hour after a feed. Infants had no respiratory symptoms for at least 3 days before measurement. Furthermore, infants were on full oral feeds, had serum electrolytes, calcium, magnesium and phosphates within normal range and did not receive any methylxanthines.

\section{ACE genotype determination}

ACE genotyping was performed on DNA extracted from whole blood, collected from an indwelling catheter or via peripheral venipuncture during routine blood sampling. The method has been previously described. ${ }^{10}$ Briefly, DNA was extracted from 200 $\mu$ l blood using Qiamp spin columns (Blood mini kit- Qiagen, QIAGEN Inc., Germantown, U.S.A). DNA was analyzed by electrophoresis on an agarose gel. DNA amplification of the $16^{\text {th }}$ ACE intron was performed using two sets of primers flanking the polymorphic site, (outer and inner primers), because mistyping of the $D / D$ genotype is reported to occur using the conventional amplification with insertion/deletion (I/D) flanking primer. ${ }^{11}$ 


\section{Measurement of PTImus}

Airflow was measured using a pneumotachograph (Mercury F10L, GM Instruments, Kilwinning, Scotland) connected to a differential pressure transducer (DP45, range \pm $2 \mathrm{~cm} \mathrm{H}_{2} \mathrm{O}$, Validyne Corp, Northridge, CA, USA). Airway pressure (Paw) was measured from a side port on the pneumotachograph using a differential pressure transducer (DP45, range $\pm 100 \mathrm{~cm} \mathrm{H} \mathrm{H}_{2} \mathrm{O}$, Validyne Corp, Northridge, CA, USA). The signals from the pressure transducers were amplified using a carrier amplifier (Validyne CD 280, Validyne Corp, Northridge, CA, USA). The pressure and flow signals were recorded and displayed in real time on a computer (Dell Optiplex GX620, Dell Inc., Texas, U.S.A) running Labview ${ }^{\mathrm{TM}}$ software (National Instruments, Austin, Texas, U.S.A) with analog-to-digital sampling at $100 \mathrm{~Hz}$ (16-bit NI PCl6036E, National Instruments, Austin, Texas, U.S.A).

$P_{0.1}$ was calculated as the airway pressure generated 100 milliseconds after an occlusion, using a face mask and a unidirectional valve which allowed expiration but not inspiration, while the infant was quietly breathing. At least four airway occlusions were performed and the average $P_{0.1}$ was calculated. Pressure-time index of the inspiratory muscles (PTImus), was calculated as: $\mathrm{PTImus}=\left(\mathrm{Pi}_{\text {mean }} / \mathrm{Pi}_{\max }\right) \times(\mathrm{Ti} / \mathrm{Ttot})$ where $\mathrm{Pi}_{\text {mean }}$ was the average airway pressure during inspiration, obtained from the formula $\mathrm{Pi}_{\text {mean }}=5 \times \mathrm{P}_{0.1} \times \mathrm{Ti}^{12} \mathrm{Pi}_{\max }$ was the maximum inspiratory airway pressure, Ti was the inspiration time and Ttot was the total time for each breath calculated from the airway flow signal. To measure $\mathrm{Pi}_{\max }$, a facemask (total deadspace, $4.5 \mathrm{~mL}$ ) was held firmly over the infant's nose and mouth and the airway was occluded at the end of a spontaneous crying effort using a unidirectional valve attached to the pneumotachograph, which allowed expiration, but not inspiration. The occlusion was 
maintained for at least four inspiratory efforts. At least three airway occlusions were performed and the maximum $\mathrm{Pi}_{\max }$ achieved for individual, was recorded.

\section{Statistical analysis}

Data was tested for normality using the Shapiro-Wilk and D'Agostino skewness tests. Differences between ACE genotype groups were assessed for statistical significance using the Kruskal-Wallis and Dunn's post-hoc non-parametric tests and Cramer's V test, as appropriate. Stepwise multiple regression analysis was performed to determine whether the polymorphism of the ACE gene is related to PTImus measurements, independently to gestational (GA) and postnatal (PNA) age, birthweight (BW), gender and support from mechanical ventilation.

Statistical analysis was performed using StatView 5.0 (SAS Institute, Inc., NC, USA), NCSS 2007 (NCSS, Utah, USA) and SPSS version 17 (SPSS Inc, Chicago, IL, USA).

\section{RESULTS}

Between January 2007 and August 2008 from the 532 infants admitted in the Neonatal Intensive Care Unit of the University of Patras, one hundred thirty two infants were included in the study. Fifty eight infants required ventilation in the initial stage of their illness. The characteristics of the study population are presented in table 1.

Eighteen infants (13.6\%) were homozygous for the I-allele (I/I), 41 (31.1\%) homozygous for the D-allele (D/D) and 73 infants (55.3\%) were heterozygous I/D. A Hardy-Weinberg equilibrium (HWE) calculation was performed to examine whether 
genotype frequencies remain constant in the studied population. Participants were in HWE (Chi square for HWE 2.613, $\mathrm{p}=0.106)$.

Overall, there were no significant differences in the characteristics of the infants with I/I, D/D and I/D ACE genotypes (table 2). Only four infants were oxygen dependent at 36 weeks postmenstrual age ( 1 infant with I/I, 2 with I/D and 1 with D/D ACE genotypes). Infants with I/I genotype had lower PTImus than infants with either D/D or I/D genotypes (Kruskal-Wallis, $p=0.000007$; Dunn's test, $z$-value $=3.58$, $p<0.01$ and $z$-value $=4.88, p<0.01$, respectively) (table 2), (figure). No difference in regards to PTImus values was found between infants with $D / D$ and I/D genotypes (Dunn's test, z-value $=1.39$, n.s). Analysis of the components of the PTImus has shown that only the ratio of $\mathrm{Pi}_{\text {mean }}$ to $\mathrm{Pi}_{\text {max }}$ was lower in infants with the $\mathrm{I} / \mathrm{I}$ genotype, compared to infants with either the $D / D$ or $I / D$ genotypes (Kruskal-Wallis, $p=$ 0.000005; Dunn's test, $z$-value $=3.33, \quad p<0.01$ and $z$-value $=4.91, \quad p<0.01$, respectively). Lower values of $\mathrm{Pi}_{\text {mean }}$ in infants with $\mathrm{I} / \mathrm{I}$ genotype compared to infants with either $D / D$ or $1 / D$ genotypes, were also observed (Kruskal-Wallis, $p=0.0009$; Dunn's test, $z$-value $=2.61, p<0.05$ and $z$-value $=3.73, p<0.01$, respectively) (table 2 ). $\mathrm{Pi}_{\text {max }}$ measurements were not statistically different between the three groups (table 2). Stepwise regression analysis revealed that ACE genotypes were significantly related to the PTImus measurements after logarithmic transformation, independent of GA, BW, PNA, gender and support from mechanical ventilation ( $p=0.005)$ (table 3).

In the subgroup of infants that never required any form of ventilatory support, 10 infants (13.5\%) were homozygous I/I, 20 (27.0\%) homozygous D/D and 44 infants (59.5\%) were heterozygous I/D. Infants in this subgroup were in HWE (Chi square for HWE 3.304, $p=0.069)$. No significant difference in the characteristics of the 
infants with I/I, D/D and I/D ACE genotypes, was found (table 4). Infants with I/I genotype had lower PTImus than infants with either D/D or I/D genotypes (KruskalWallis, $p=0.0022 ;$ Dunn's test, $z$-value $=3.07, p<0.01$ and $z$-value $=3.39, p<0.01$, respectively), (table 4). No difference regarding PTImus values was found between infants with D/D and I/D genotypes (Dunn's test, z-value $=0.0008$, n.s). The ratio of $\mathrm{Pi}_{\text {mean }}$ to $\mathrm{Pi}_{\text {max }}$ was lower in infants with the $\mathrm{I} / \mathrm{I}$ genotype, compared to infants with either the $D / D$ or I/D genotypes (Kruskal-Wallis, $p=0.0015$; Dunn's test, $z$-value= 2.95, $\mathrm{p}<0.01$ and $z$-value $=3.58, \mathrm{p}<0.01$, respectively). $\mathrm{Pi}_{\max }$ measurements were not statistically different between the three groups (table 4), but $\mathrm{Pi}_{\text {mean }}$ was lower in infants with $\mathrm{I} / \mathrm{I}$ genotype, compared to infants with either $\mathrm{D} / \mathrm{D}$ or $\mathrm{I} / \mathrm{D}$ genotypes (Kruskal-Wallis, $p=0.003$; Dunn's test, $z$-value $=3.31, p<0.01$ and $z$-value $=3.00$, $p<0.01$, respectively). In this subgroup, forty six infants never required any oxygen supplementation; 6 infants (13.0\%) were homozygous I/I, $13(28.3 \%)$ homozygous D/D and 27 infants (58.7\%) were heterozygous I/D. Infants in the subgroup that never required any oxygen supplementation were in HWE (Chi square for HWE 1.872, $\mathrm{p}=0.171$ ). The median (range) of PTImus of the whole subgroup was 0.078 (0.028-0.119). Infants with I/I ACE genotype had lower PTImus than infants with either $\mathrm{D} / \mathrm{D}$ or $\mathrm{I} / \mathrm{D}$ genotypes [median (range), $0.040(0.028-0.083)$ versus 0.073 (0.052-119) and 0.080 (0.040-117), respectively; Kruskal-Wallis, $p=0.0075$; Dunn's test, $z$-value $=2.55, p<0.05$ and $z$-value $=3.11, p<0.01$, respectively]. The ratio of $\mathrm{Pi}_{\text {mean }}$ to $\mathrm{Pi}_{\text {max }}$ and $\mathrm{Pi}_{\text {mean }}$ were lower in infants with the $\mathrm{I} / \mathrm{I}$ ACE genotype, compared to infants with either D/D or I/D genotypes (Kruskal-Wallis, $p=0.0047$; Dunn's test, zvalue $=2.42, p<0.05$ and $z$-value $=3.27, p<0.01$, respectively and Kruskal-Wallis, $p=$ 0.019; Dunn's test, $z$-value $=2.46, p<0.05$ and $z$-value $=2.74, p<0.05$, respectively). 
In the subgroup of the infants who required ventilation in the initial stage of their illness, 8 infants (13.8\%) were homozygous I/I, 21 (36.2\%) homozygous D/D and 29 infants (50\%) were heterozygous I/D. Participants were in HWE (Chi square for HWE 0.1622, $p=0.687$ ). Infants with I/I ACE genotype had lower PTImus than infants with either $D / D$ or I/D genotypes (Kruskal-Wallis, $p=0.0024$; Dunn's test, $z$-value $=1.98$, $\mathrm{p}<0.05$ and $\mathrm{z}$-value $=3.37, \mathrm{p}<0.01$, respectively)

\section{DISCUSSION}

In this study, an association between ACE genotypes and PTImus measurements in infants, was demonstrated. Infants homozygous for the I-allele had lower PTImus values than infants homozygous for the D-allele and heterozygous I/D. No difference in regards to PTImus values was found between infants with D/D and I/D genotypes. Furthermore, the ACE polymorphism was related to the PTImus measurements, independent of other factors, which could affect respiratory muscle performance. The association between ACE genotypes and PTImus measurements was replicated on the subgroup of infants that required ventilation in the initial stage of their illness and on the subgroup of infants that never required any form of ventilatory support.

One limitation of our study was the fact that measurements in an age-matched control group, consisting of infants in the postnatal ward, were not performed. The recruitment of such babies is extremely difficult. To overcome this issue, we measured infants in the neonatal unit that are free of factors that could possibly interfere with respiratory muscle function such as requirement of respiratory support. 
One part of the whole population consisted of infants that never required oxygen supplementation, indeed, and a separate analysis was performed in this subgroup.

Fatigue of respiratory muscles may result in an inability to maintain adequate alveolar ventilation and respiratory failure. A simple method of studying the endurance properties of inspiratory muscles, is the measurement of the duration that a specific submaximal task (maximum sustainable ventilation, external mechanical load) can be maintained (time limit or endurance time, TLim), referred to as the point where the inspiratory muscles failed to maintain the task, despite maximal effort. ${ }^{7}$ However, this kind of test is difficult to be applied to infants. Diaphragmatic pressuretime index (PTIdi), the product of the ratio of mean inspiratory transdiaphragmatic pressure per breath $\left(\mathrm{Pdi}_{\text {mean }}\right)$ to maximum transdiaphragmatic pressure $\left(\mathrm{Pdi}_{\max }\right)$ and the ratio of the inspiratory time ( $\mathrm{Ti}$ ) to the total respiratory cycle time (Ttot), is closely related to the Tlim. ${ }^{13}$ The determination of PTIdi, however, is rather invasive, since it requires the placement of an esophageal catheter. Analysis of inspiratory muscle function by measurement of PTImus was first described by Gaultier et al. ${ }^{12}$ As measurement of $P_{01}$ is non-invasive, measurement of PTImus has the advantage of being relatively easy to be performed. This index was validated in adults. ${ }^{8}$ Studies in healthy children, demonstrated that PTImus ranged between 0.036 and $0.090 .{ }^{14,15}$ In spontaneously breathing infants, PTImus was compared to PTIdi and an agreement between the two methods, using Bland and Altman analysis, was found. ${ }^{9}$ In this study, PTImus measurements in infants that never required any oxygen supplementation ranged between 0.028 and 0.119 , something that is close to the values observed in healthy children. 
Several studies have suggested that there is an association of the ACE I/D polymorphism with exercise performance. British Army recruits were examined before and after an identical 10-week general physical training program. ${ }^{5}$ Although, pre-training performance was independent of genotype, the improvement in performance was 11 -fold greater among those of $\mathrm{I} / \mathrm{I}$, compared with $\mathrm{D} / \mathrm{D}$ genotype. ${ }^{5}$ Consistently, a significant excess of the $\mathrm{l} / \mathrm{I}$ genotype was found in Australian national rowers attending their pre-Olympics selection trial, compared to matched healthy controls. ${ }^{16}$ The frequency of I-allele was higher in British Olympic-standard distance runners. ${ }^{4}$ Furthermore, genotype distribution and I-allele frequency differed significantly between elite mountaineers, who had ascended beyond $7000 \mathrm{~m}$ without the use of supplemental oxygen and controls. ${ }^{5}$ All such studies are prone to difficulties in comparison, as the definition of the "elite athlete" and the heterogeneity of the elite athlete phenotype may be variable. The polymorphism of the ACE gene has been examined in infants, but not in association with muscle endurance. ${ }^{17,18}$

The association of the ACE genotype and respiratory muscle function, has not been widely examined yet. In a study of COPD (Chronic Obstructive Pulmonary Disease) patients and healthy control subjects, the ACE genotype was related to the regulation of skeletal muscle aerobic work efficiency, but it was not associated with the ventilatory responses to exercise. ${ }^{19}$ The relationship between circulating ACE activity, ACE I/D genotype, mechanical efficiency and the maximal rate of oxygen uptake has been assessed in untrained women. ${ }^{20}$ Neither circulating ACE activity nor ACE genotype showed a statistically significant association with any of the endurance phenotypes measured. However, ACE inhibition in heart failure increases respiratory muscle strength ${ }^{21}$ and exercise tolerance..$^{22,23}$ 
The enhanced endurance performance in the ACE I/I genotype may be due to higher oxygen delivery, conversion of fast-twitch, type II, to slow-contracting, type I, muscle fibers, increased mitochondrial numbers and density, and raised myoglobin content of the muscle. ${ }^{24}$ ACE I-allele compared to D-allele is associated with an increased percentage of fatigue-resistant type I skeletal muscle fibres ${ }^{25}$ and hence with enhanced mechanical ${ }^{6}$ and metabolic $^{26}$ efficiency of the trained muscle. The effect of ACE genotype on energy balance, might be mediated through several different pathways, such as systemic endocrine or nutritional mechanisms. ${ }^{26}$ Local RASs exist in several tissues. The adipose RAS may alter mobilization of substrate from fat stores and affect metabolism. ${ }^{26}$ Finally, the local skeletal muscle RAS may modify the use of substrate and enhance the metabolic efficiency of the muscles. ${ }^{26}$

Several factors may affect respiratory muscle endurance such as nutrition, ${ }^{27}$ prolonged ventilatory support, ${ }^{28}$ drugs, ${ }^{29,30,31}$ as well as phosphate, ${ }^{32}$ calcium $^{33}$ and magnesium ${ }^{34}$ blood levels. All infants were measured prior to discharge, being on full enteral feeds, they did not receive any medication and their biochemistry blood tests were within the normal range during measurements.

PTImus integrates all components that may affect inspiratory muscles while breathing at rest, i.e, timing of breathing cycle, inspiratory demand at rest and maximal inspiratory force reserve. When we considered these components, the timing of the breath cycle (Ti/Ttot) and the maximal inspiratory force reserve $\left(\mathrm{Pi}_{\max }\right)$ were not statistically different between infants with I/I, D/D and I/D ACE genotype. However, the $\mathrm{Pi}_{\text {mean }} / \mathrm{Pi}_{\text {max }}$ ratio and the $\mathrm{Pi}_{\text {mean }}$ (inspiratory demand at rest), were lower in infants with the $\mathrm{I} / \mathrm{I}$ compared with infants with $\mathrm{D} / \mathrm{D}$ and $\mathrm{I} / \mathrm{D}$ genotypes. $A$ higher inspiratory demand at rest $\left(\mathrm{Pi}_{\text {mean }}\right)$ in infants with $\mathrm{D} / \mathrm{D}$ and $\mathrm{I} / \mathrm{D}$, compared with 
infants with I/I ACE genotypes, could suggest that a higher elastic and/or resistive load (existing lung disease) imposed on the respiratory muscles of infants with I/D and D/D genotypes compared with infants with I// ACE genotypes, may exist. Indeed, infants with non-I/I ACE genotypes have been suggested to be more likely to develop bronchopulmonary dysplasia, perhaps as a consequence of the role of ACE activity in the inflammatory cascade. ${ }^{17}$ However, in this study, no difference in regards to bronchopulmonary dysplasia rate between infants with I/I, I/D and D/D ACE genotypes was noted. In addition, the same association between ACE genotypes and $\mathrm{Pi}_{\text {mean }}$ was replicated in a subgroup of infants that never required any form of respiratory support and in infants that never required any oxygen supplementation.

Fatigue threshold of inspiratory muscles has not yet been established in infants. All infants measured, were spontaneously breathing on air. Therefore, the PTImus results of all infants would be expected to lie far from the fatigue threshold. These results, however, indicate an increased effectiveness of inspiratory muscles of infants with the I// ACE genotype, due to a lower energy demand and thus to a lower risk of respiratory muscle fatigue, under conditions that increase inspiratory load. ${ }^{35,36}$ The findings of this study suggest that in clinical practice, infants with $1 / /$ ACE genotype, if supported by mechanical ventilation, may succeed extubation more often than infants with I/D and D/D ACE genotypes or may have a lower risk of respiratory failure under conditions that increase inspiratory load, such as respiratory infections.

In conclusion, these results suggest that an association of ACE genotypes with 
PTImus measurements may exist in infants, independent of other factors, which could affect respiratory muscle function.

\section{ACKNOWLEDGMENTS}

None

\section{COMPETING INTERESTS}

The authors declare that they have no financial or other relationships that might lead to a conflict of interest.

\section{FUNDING}

None

\section{ETHICS COMMITTEE APPROVAL}

The study was approved by the Ethics Committee of the University Hospital of Patras, Patras, Greece 


\section{REFERENCES}

1. Woods DR, Pollard AJ, Collier DJ, Jamshidi Y, Vassiliou V, Hawe E, Humphries SE, Montgomery HE. Insertion/deletion polymorphism of the angiotensin I-converting enzyme gene and arterial oxygen saturation at high altitude. Am J Respir Crit Care Med 2002;166(3):362-366.

2. Jones A, Woods DR. Skeletal muscle RAS and exercise performance. Int J Biochem Cell Biol 2003;35(6):855-866.

3. Tiret L, Rigat B, Visvikis S, Breda C, Corvol P, Cambien F, Soubrier F. Evidence, from combined segregation and linkage analysis, that a variant of the angiotensin l-converting enzyme (ACE) gene controls plasma ACE levels. Am J Hum Genet 1992;51(1):197-205.

4. Myerson S, Hemingway H, Budget R, Martin J, Humphries S, Montgomery $\mathrm{H}$. Human angiotensin I-converting enzyme gene and endurance performance. J Appl Physiol 1999;87(4):1313-1316.

5. Montgomery HE, Marshall R, Hemingway $H$, Myerson S, Clarkson P, Dollery C, Hayward M, Holliman DE, Jubb M, World M, Thomas EL, Brynes AE, Saeed N, Barnard M, Bell JD, Prasad K, Rayson M, Talmud PJ, Humphries SE. Human gene for physical performance. Nature 1998;393(6682):221-222.

6. Williams AG, Rayson MP, Jubb M, World M, Woods DR, Hayward M, Martin J, Humphries SE, Montgomery HE. The ACE gene and muscle performance. Nature 2000;403(6770):614.

7. ATS/ERS Statement on respiratory muscle testing. Am J Respir Crit Care Med 2002;166(4):518-624.

8. Ramonatxo M, Boulard P, Prefaut C. Validation of a noninvasive tension-time index of inspiratory muscles. J Appl Physiol 1995;78(2):646-653. 
9. Dimitriou G, Patel S, Patel J, Rafferty GF, Greenough A. A non-invasive tension-time index of respiratory muscles in neonates. European Respiratory Society Annual Congress. Glasgow, UK; 2004.

10. Lindpaintner K, Pfeffer MA, Kreutz R, Stampfer MJ, Grodstein F, LaMotte F, Buring J, Hennekens $\mathrm{CH}$. A prospective evaluation of an angiotensinconverting-enzyme gene polymorphism and the risk of ischemic heart disease. N Engl J Med 1995;332(11):706-711.

11. Shanmugam V, Sell KW, Saha BK. Mistyping ACE heterozygotes. PCR Methods Appl 1993;3(2):120-121.

12. Gaultier C, Boule M, Tournier G, Girard F. Inspiratory force reserve of the respiratory muscles in children with chronic obstructive pulmonary disease. Am Rev Respir Dis 1985;131(6):811-815.

13. Bellemare F, Grassino A. Effect of pressure and timing of contraction on human diaphragm fatigue. J Appl Physiol 1982;53(5):1190-1195.

14. Hayot M, Guillaumont S, Ramonatxo M, Voisin M, Prefaut C. Determinants of the tension-time index of inspiratory muscles in children with cystic fibrosis. Pediatr Pulmonol 1997;23(5):336-343.

15. Hahn A, Ankermann T, Claass A, Mann M, Lindemann H, Neubauer BA. Noninvasive tension time index in relation to severity of disease in children with cystic fibrosis. Pediatr Pulmonol 2008;43(10):973-981.

16. Gayagay G, Yu B, Hambly B, Boston T, Hahn A, Celermajer DS, Trent RJ. Elite endurance athletes and the ACE I allele--the role of genes in athletic performance. Hum Genet 1998;103(1):48-50. 
17. Kazzi SN, Quasney MW. Deletion allele of angiotensin-converting enzyme is associated with increased risk and severity of bronchopulmonary dysplasia. J Pediatr 2005;147(6):818-822.

18. Harding D, Dhamrait S, Marlow N, Whitelaw A, Gupta S, Humphries S, Montgomery $\mathrm{H}$. Angiotensin-converting enzyme DD genotype is associated with worse perinatal cardiorespiratory adaptation in preterm infants. J Pediatr 2003;143(6):746-749.

19. Zhang X, Wang C, Dai H, Lin Y, Zhang J. Association between angiotensinconverting enzyme gene polymorphisms and exercise performance in patients with COPD. Respirology 2008;13(5):683-688.

20. Day SH, Gohlke P, Dhamrait SS, Williams AG. No correlation between circulating ACE activity and VO2max or mechanical efficiency in women. Eur J Appl Physiol 2007;99(1):11-18.

21. Coirault C, Hagege A, Chemla D, Fratacci MD, Guerot C, Lecarpentier Y. Angiotensin-converting enzyme inhibitor therapy improves respiratory muscle strength in patients with heart failure. Chest 2001;119(6):1755-1760.

22. Mancini DM, Davis L, Wexler JP, Chadwick B, LeJemtel TH. Dependence of enhanced maximal exercise performance on increased peak skeletal muscle perfusion during long-term captopril therapy in heart failure. Journal of the American College of Cardiology 1987;10(4):845-850.

23. Drexler $H$, Banhardt $U$, Meinertz $T$, Wollschlager $H$, Lehmann $M$, Just $H$. Contrasting peripheral short-term and long-term effects of converting enzyme inhibition in patients with congestive heart failure. A double-blind, placebocontrolled trial. Circulation 1989;79(3):491-502. 
24. Bloom SR, Johnson RH, Park DM, Rennie MJ, Sulaiman WR. Differences in the metabolic and hormonal response to exercise between racing cyclists and untrained individuals. J Physiol 1976;258(1):1-18.

25. Zhang B, Tanaka H, Shono N, Miura S, Kiyonaga A, Shindo M, Saku K. The I allele of the angiotensin-converting enzyme gene is associated with an increased percentage of slow-twitch type I fibers in human skeletal muscle. Clin Genet 2003;63(2):139-144.

26. Montgomery H, Clarkson P, Barnard M, Bell J, Brynes A, Dollery C, Hajnal J, Hemingway H, Mercer D, Jarman P, Marshall R, Prasad K, Rayson M, Saeed N, Talmud $\mathrm{P}$, Thomas L, Jubb M, World M, Humphries S. Angiotensinconverting-enzyme gene insertion/deletion polymorphism and response to physical training. Lancet 1999;353(9152):541-545.

27. Arora NS, Rochester DF. Effect of body weight and muscularity on human diaphragm muscle mass, thickness, and area. J Appl Physiol 1982;52(1):6470.

28. Tobin MJ, Alex C. Discontinuation of mechanical ventilation. In: Tobin M, editor. Principles and Practice of Mechanical Ventilation. New York: McGrawHill; 1994. p 1177-1206.

29. Aubier M, Murciano D, Menu Y, Boczkowski J, Mal H, Pariente R. Dopamine effects on diaphragmatic strength during acute respiratory failure in chronic obstructive pulmonary disease. Ann Intern Med 1989;110(1):17-23.

30. Vires N, Aubier M, Murciano D, Fleury B, Talamo C, Pariente R. Effects of aminophylline on diaphragmatic fatigue during acute respiratory failure. Am Rev Respir Dis 1984;129(3):396-402. 
31. Supinski GS, Levin S, Kelsen SG. Caffeine effect on respiratory muscle endurance and sense of effort during loaded breathing. J Appl Physiol 1986;60(6):2040-2047.

32. Aubier M, Murciano D, Lecocguic $Y$, Viires N, Jacquens $Y$, Squara $P$, Pariente R. Effect of hypophosphatemia on diaphragmatic contractility in patients with acute respiratory failure. N Engl J Med 1985;313(7):420-424.

33. Aubier M, Viires N, Piquet J, Murciano D, Blanchet F, Marty C, Gherardi R, Pariente R. Effects of hypocalcemia on diaphragmatic strength generation. J Appl Physiol 1985;58(6):2054-2061.

34. Dhingra S, Solven F, Wilson A, McCarthy DS. Hypomagnesemia and respiratory muscle power. Am Rev Respir Dis 1984;129(3):497-498.

35. Roussos C, Macklem P. Inspiratory muscle fatigue. In: Macklem P, Mead J, editors. The Handbook of Physiology The Respiratory System. Volume III. Bethesda, MD: American Physiological Society; 1986. p 511-527.

36. Vassilakopoulos T, Roussos C. Neuromuscular respiratory failure. In: Slutsky A, Takala R, Torres R, editors. Clinical Critical Care Medicine. St. Louis: Mosby; 2006. p 275-282. 
Figure legend

Comparison of PTImus results of infants with I/I $(n=18), I / D(n=73)$ and D/D ( $n=41)$ ACE genotypes. Box and whisker plot: fine horizontal lines represent $10^{\text {th }}, 25^{\text {th }}, 50^{\text {th }}$, $75^{\text {th }}$ and $90^{\text {th }}$ centiles of PTImus. Outliers are plotted as discrete data points. 